\section{Whole exome sequencing reveals NOTCH1 mutations in anaplastic large cell lymphoma and points to Notch both as a key pathway and a potential therapeutic target}

\begin{abstract}
Hugo Larose, ${ }^{1,2}$ Nina Prokoph, ${ }^{1,2}$ Jamie D. Matthews, ${ }^{1}$ Michaela Schlederer, ${ }^{3}$ Sandra Högler, ${ }^{4}$ Ali F. Alsulami, ${ }^{5}$ Stephen P. Ducray, ${ }^{1,2}$ Edem Nuglozeh, ${ }^{6}$ Mohammad Feroze Fazaludeen, ${ }^{7}$ Ahmed Elmouna, ${ }^{6}$ Monica Ceccon, ${ }^{2,8}$ Luca Mologni, ${ }^{2,8}$ Carlo Gambacorti-Passerini, ${ }^{2,8}$ Gerald Hoefler, ${ }^{9}$ Cosimo Lobello, ${ }^{2,10}$ Sarka Pospisilova, ${ }^{2,10,11}$ Andrea Janikova, ${ }^{2,11}$ Wilhelm Woessmann, ${ }^{2,12}$ Christine Damm-Welk, ${ }^{2,12}$ Martin Zimmermann, ${ }^{13}$ Alina Fedorova, ${ }^{14}$ Andrea Malone,${ }^{15}$ Owen Smith, ${ }^{15}$ Mariusz Wasik, ${ }^{2,16}$ Giorgio Inghirami, ${ }^{17}$ Laurence Lamant, ${ }^{18}$ Tom L. Blundell, ${ }^{5}$ Wolfram Klapper, ${ }^{19}$ Olaf Merkel, ${ }^{2,3}$ G. A. Amos Burke, ${ }^{20}$ Shahid Mian, ${ }^{6}$ Ibraheem Ashankyty, ${ }^{21}$ Lukas Kenner ${ }^{2,3,22}$ and Suzanne D. Turner ${ }^{1,2,10}$
\end{abstract}

${ }^{1}$ Department of Pathology, University of Cambridge, Cambridge, UK; ${ }^{2}$ European Research Initiative for ALK Related Malignancies (ERIA; www.ERIALCL.net); ${ }^{3}$ Department of Pathology, Medical University of Vienna, Vienna, Austria; ${ }^{4}$ Unit of Laboratory Animal Pathology, University of Veterinary Medicine Vienna, Vienna, Austria; ${ }^{5}$ Department of Biochemistry, University of Cambridge, Tennis Court Road, Cambridge, UK; ${ }^{6}$ Molecular Diagnostics and Personalised Therapeutics Unit, Colleges of Medicine and Applied Medical Sciences, University of Ha'il, Ha'il, Saudi Arabia; ${ }^{7}$ Neuroinflammation Research Group, Department of Neurobiology, A.I Virtanen Institute for Molecular Sciences, University of Eastern Finland, Finland; ${ }^{8}$ University of Milano-Bicocca, Monza, Italy ; ${ }^{9}$ Diagnostic and Research Institute of Pathology, Medical University of Graz, Graz, Austria ; ${ }^{10}$ Center of Molecular Medicine, CEITEC, Masaryk University, Brno, Czech Republic; ${ }^{11}$ Department of Internal Medicine - Hematology and Oncology, University Hospital Brno, Czech Republic; ${ }^{12}$ University Hospital Hamburg-Eppendorf, Pediatric Hematology and Oncology, Hamburg, Germany; ${ }^{13}$ Department of Pediatric Hematology/Oncology and Blood Stem Cell Transplantation, Hannover Medical School, Hannover, Germany; ${ }^{14}$ Belarusian Center for Pediatric Oncology, Hematology and Immunology, Minsk, Belarus; ${ }^{15}$ Our Lady's Children's Hospital, Crumlin, Ireland; ${ }^{16}$ Perelman School of Medicine, Philadelphia, PA, USA; ${ }^{17}$ Department of Pathology and Laboratory Medicine, Cornell University, New York, NY USA; ${ }^{18}$ Institut Universitaire du Cancer Toulouse, Oncopole et Université Paul-Sabatier, Toulouse, France; ${ }^{19}$ Department of Pathology, Hematopathology Section, UKSH Campus Kiel, Kiel, Germany; ${ }^{20}$ Department of Paediatric Oncology, Addenbrooke's Hospital, Cambridge, UK; ${ }^{21}$ Department of Medical Technology Laboratory, College of Applied Medical Sciences, King Abdulaziz University, Jeddah, Saudi Arabia and ${ }^{22}$ Ludwig-Boltzmann Institute for Cancer Research, Vienna, Austria

\section{ABSTRACT}

$\mathrm{P}$ atients diagnosed with anaplastic large cell lymphoma (ALCL) are still treated with toxic multi-agent chemotherapy and as many as $25-50 \%$ of patients relapse. To understand disease pathology and to uncover novel targets for therapy, we performed whole-exome sequencing of anaplastic lymphoma kinase (ALK) ALCL, as well as gene-set enrichment analysis. This revealed that the T-cell receptor and Notch pathways were the most enriched in mutations. In particular, variant T349P of NOTCH1, which confers a growth advantage to cells in which it is expressed, was detected in $12 \%$ of $\mathrm{ALK}^{+}$and $\mathrm{ALK}^{-}$ALCL patients' samples. Furthermore, we demonstrated that NPM-ALK promotes NOTCH1 expression through binding of STAT3 upstream of NOTCH1. Moreover, inhibition of NOTCH1 with $\gamma$ secretase inhibitors or silencing by short hairpin RNA leads to apoptosis; cotreatment in vitro with the ALK inhibitor crizotinib led to additive/synergistic antitumor activity suggesting that this may be an appropriate combination
Haematologica 2021

Volume 106(6):1693-1704

\section{Correspondence:}

SUZANNE D. TURNER

sdt36@cam.ac.uk

Received: September 19, 2019

Accepted: April 9, 2020

Pre-published: April 23, 2020.

https://doi.org/10.3324/haematol.2019.238766

(C)2021 Ferrata Storti Foundation

Material published in Haematologica is covered by copyright. All rights are reserved to the Ferrata Storti Foundation. Use of published material is allowed under the following terms and conditions:

https://creativecommons.org/licenses/by-nc/4.0/legalcode. Copies of published material are allowed for personal or internal use. Sharing published material for non-commercial purposes is subject to the following conditions:

https://creativecommons.org/licenses/by-nc/4.0/legalcode, sect. 3. Reproducing and sharing published material for commercial purposes is not allowed without permission in writing from the publisher. 
therapy for future use in the circumvention of ALK inhibitor resistance. Indeed, crizotinib-resistant and -sensitive ALCL were equally sensitive to $\gamma$-secretase inhibitors. In conclusion, we show a variant in the extracellular domain of NOTCH1 that provides a growth advantage to cells and confirm the suitability of the Notch pathway as a second-line druggable target in ALK+ ALCL.

\section{Introduction}

Systemic anaplastic large cell lymphoma (ALCL) is a Tcell malignancy accounting for approximately $15 \%$ of all cases of pediatric lymphoma and $1-2 \%$ of adult lymphomas. ${ }^{1}$ The majority of pediatric cases $(>90 \%)$ express NPM-ALK, the result of the $t(2 ; 5)(p 23 ; q 35)$ translocation, creating a fusion between the nucleolar phosphoprotein gene nucleophosmin1 (NPM1) and anaplastic lymphoma kinase $(A L K)$, leading to the $\mathrm{ALK}^{+} \mathrm{ALCL}$ diagnostic entity, although other ALK fusion proteins also exist., ${ }^{2,3}$ ALK fusion proteins induce the activation of several downstream signaling pathways involved in oncogenesis, including PI3K, ERK1/2 MAP kinase, and JAK-STAT.

Genetic studies conducted to date have not revealed consistent genetic abnormalities among $\mathrm{ALK}^{+} \mathrm{ALCL}$, although a higher frequency of genomic gains and losses has been associated with a poorer prognosis. ${ }^{4-6}$ Genomic gains include the region encoding the NOTCH1 locus, ${ }^{4}$ which may explain why NOTCH1 is expressed in the majority of $\mathrm{ALK}^{+} \mathrm{ALCL}$ and in some ALK $\mathrm{ALCL}^{-7,8}$ In contrast, for $\mathrm{ALK}^{-}$ ALCL, mutations in the JAK1/STAT3 pathway have been described. ${ }^{9}$

$\mathrm{ALK}^{+} \mathrm{ALCL}$ is largely a chemosensitive malignancy, although despite good initial responses, the relapse rate reaches $50 \%$ independently of the chemotherapy regimen used; $;^{10-14}$ therefore, new therapies are needed for patients who do not respond to standard chemotherapy. There is also a need for less toxic treatment schedules for low-risk patients. Crizotinib, a small molecule ATP-competitive inhibitor of ALK/MET/ROS1 which is currently in clinical trials (NCT01606878, NCT02034981) for pediatric ALK ${ }^{+}$ lymphoid malignancies, given as monotherapy produces remission in more than $80 \%$ of relapsed patients, although rapid relapse on discontinuation of therapy has been reported. ${ }^{11,15-17}$ As such, second-line treatment and combination therapies for relapsed patients are still required. We therefore conducted whole exome sequencing (WES) of $25 \mathrm{ALK}^{+}$ ALCL tumors, validating data in a further 78 cases of ALCL to understand disease pathology and to uncover novel targets for therapy. Beside the T-cell receptor (TCR) pathway, the most commonly affected signaling axis is that involving NOTCH1, of which a T349P variant provides a growth advantage to cells. Furthermore, NOTCH1 is expressed as a consequence of NPM-ALK-driven STAT3 activity, a key signaling node in ALCL. Accordingly, the NOTCH1 pathway provides a target for second-line therapy, whereby $\gamma$-secretase inhibitors (GSI) show synergistic activity with inhibitors of ALK, and are efficacious as single agents in ALK-inhibitor resistant cell lines. Finally, we show evidence that NOTCH1 is a biomarker predictive of relapse risk.

\section{Methods}

\section{Patients' samples}

Patients' tumor tissues (at initial presentation) and matched peripheral blood DNA were obtained following patient/parental consent according to both the Declaration of Helsinki and local guidelines from the following institutions: Children's Cancer and Leukaemia Group tissue bank, Newcastle, UK (Online Supplementary Table S1); Institut Universitaire du Cancer Toulouse, France; University Hospital Brno, Czech Republic; Biobank of the Medical University of Graz, Austria; Belarusian Center for Pediatric Oncology, Hematology and Immunology, Minsk, Belarus; Justus-Liebig University, Giessen, Germany; and Our Lady's Children's Hospital, Crumlin, Ireland (Online Supplementary Table S2). All tissues were obtained and processed with full ethical approval (NHS Research ethics committee reference numbers 07/O0104/16, 06/MRE04/90 and 08/H0405/22+5).

The patient tissue microarray used here has been described elsewhere. ${ }^{18}$ Briefly, formalin-fixed paraffin-embedded (FFPE) tissue specimens from pediatric, patients with NPM-ALK ${ }^{+}$ALCL treated in the Berlin-Frankfurt-Munster (BFM) group study NHL-BFM90, NHL-BFM95 or patients enrolled in the European intergroup trial ALCL99 between August 1998 and December 2008 were obtained from both male and female children (Online Supplementary Table S3) with informed consent and in accordance with the Declaration of Helsinki. Eligibility was confirmed by demonstration of NPMALK positivity of the tumor either by NPM-ALK polymerase chain reaction, two-color fluorescence in situ hybridization for the $\mathrm{t}(2 ; 5)$, or nuclear and cytoplasmic staining for ALK. The inclusion criteria were fulfilled by 89 patients.

\section{Whole exome sequencing}

DNA was extracted from fresh-frozen tissue from patients $(n=18$; with a tumor content $>90 \%$ ) and matched peripheral blood from four patients using the OIAgen DNAeasy Blood and Tissue Kit (Qiagen, Hilden, Germany), following the manufacturer's instructions. Library preparation was conducted using the Nextera Rapid Capture Exome Kit before samples were sequenced, at either the Washington State University Core (Illumina HiSeq2500) or the Molecular Diagnostics and Personalized Therapeutics Unit, University of Ha'il (Illumina MiSeq) (Online Supplementary Table S4). Sequencing data are available at the Sequence Read Archive (https://www.ncbi.nlm.nih.gov/sra) under accession number PRJNA491296. The bioinformatic processing is detailed in the Online Supplementary Methods.

\section{Immunohistochemistry, pathology and quantification}

Immunohistochemistry was performed on FFPE sections with the conventional avidin-biotin-peroxidase method. Heat antigen retrieval was performed using citrate buffer, $\mathrm{pH}$ 6.1. Endogenous peroxidases were quenched by incubating sections in $3 \% \mathrm{H}_{2} \mathrm{O}_{2}$ in phosphate-buffered saline (PBS) for $10 \mathrm{~min}$. An avidin/biotin and a protein block were subsequently performed. Primary antibodies (Online Supplementary Table S5) were added in 1\% bovine serum albumin/PBS at $4^{\circ} \mathrm{C}$ overnight. Slides were incubated with biotinconjugated secondary antibodies and then with horse radish peroxidase (HRP) using the IDtect Super Stain System - HRP and developed under visual control using aminoethyl carbazole. Hemalaun counterstaining was performed and slides mounted with AquaTex. Sections were washed with PBS three times in between each step. Stained slides were assessed for cleaved NOTCH1 staining by an experienced pathologist (blinded with respect to clinicopathological parameters and patients' outcome) 
using the histoscore system. Stained slides were scored qualitatively for the intensity of staining and classified as showing negative, weak, moderate or strong staining (to qualify for 'moderate' or 'strong' staining, at least $10 \%$ of cells had to stain positive). Analysis of event-free survival was performed as described previously, ${ }^{19}$ grouping negative and weak staining into 'low cleaved NOTCH1 expression' and moderate and strong into 'high cleaved NOTCH1 expression'.

\section{Statistical analyses}

All experiments were executed in biological triplicates. The MTT, RealTimeGlo, apoptosis, cell cycle and quantitative polymerase chain reaction assays were additionally executed with technical triplicates. All plots are representative of the mean of the biological replicates, while the error bars represent the standard deviation. Two-tailed $t$-tests were used to calculate the $P$-value when comparing two samples (multiple comparisons were corrected using the Holm-Sidak method); when comparing more than two samples, two-way analysis of variance (ANOVA) was used (again, multiple comparisons were corrected using the HolmSidak method). Statistical tests were conducted using GraphPad PRISM 8 (Graphpad).

\section{Results}

The genomic profile of anaplastic large cell lymphoma

Eighteen ALK ${ }^{+}$ALCL exomes sequenced in this study in addition to seven previously sequenced samples ${ }^{9}$ (Online Supplementary Table S1) were analyzed. The samples comprised 17 pediatric cases ( $\leq 18$ years) and eight adult cases (Online Supplementary Table S4); a flowchart illustrating the cohorts of patients is shown in Online Supplementary Figure S1. All patient samples were collected at diagnosis. Data regarding variants found in at least a quarter of the patients are summarized in Figure 1, which shows that the most commonly mutated genes in both adult and pediatric cases were TYW1B, DEFB132 and KCNJ18 (the full list of variants can be found in Online Supplementary Table S6). None of the variants in these genes has been reported previously in hematologic malignancies and were not studied further here. Two patients presented with one mutation each in TP53 (COSMIC ID: COSM3958801 and COSM9969). We also studied copy number variations, but found no novel events larger than 100,000 bp present consistently in more than one sample at a sequencing depth of at least $50 \mathrm{x}$ (Online Supplementary Figure S2A, Online Supplementary Table S7), as previously observed. ${ }^{4}$ Among previously reported alterations in ALCL, a single copy gain on chromosome 7 was observed in three patient tumor samples $\left(\mathrm{S} 3, \mathrm{~S} 9\right.$ and S15) ${ }^{5}$ and a single copy loss on chromosome $17 p$ was also seen in three patients (S9, S14 and S57). ${ }^{6}$

\section{The most predominant single nucleotide variants are non-synonymous and are present at higher levels in patients who subsequently relapsed}

The majority of somatic variants detected in the 25 tumor samples were non-synonymous single nucleotide variants $(39.4 \%)$, in keeping with a previous publication reporting that the $\mathrm{ALK}^{+}$ALCL genome is largely stable. ${ }^{4}$ Single nucleotide variants were followed in frequency by frameshift and non-frameshift deletions and splice variants $(24.1 \%, 10.8 \%$ and $10.6 \%$, respectively), while the germline genome of ALCL patients points to an overwhelming presence of single nucleotide polymorphisms
(89.3\%) (Online Supplementary Figure S2B). The proportion of each type of variant detected differed between patient tumors (Figure $2 \mathrm{~B}$ ), although in general, pediatric patients known to have relapsed $(n=9)$ had a significantly higher proportion of non-synonymous single nucleotide variants than patients who did not $(\mathrm{n}=9 ; P<0.0001)$ (Figure $2 \mathrm{~B}$ ), suggesting that a high percentage of non-synonymous single nucleotide variants at diagnosis may be indicative of relapse, although this requires validation in a larger dataset of patients treated with comparable treatment protocols.

\section{Deficiency of DNA repair mechanisms and spontaneous deamination of 5-methyl cytosine are identified as signatures of anaplastic large cell lymphoma}

Online Supplementary Figure S2C shows the prevalence, in representative patient $S 57$, of the 96 variant types that were used to derive the mutational signatures (Online Supplementary Figure S2D). Examining the type of mutations in the patients for whom matched peripheral blood was available $(\mathrm{n}=11)$, showed an enrichment for mutational signatures $1,3,12$ and $26^{20}$ (Figure $2 \mathrm{C}$ ). Interestingly, $1 \mathrm{~A}$ is a signature based on the prevalence of $\mathrm{C}>\mathrm{T}$ transitions at $\mathrm{NpCpG}$ trinucleotides and is associated with spontaneous deamination of 5 -methyl-cytosine ${ }^{21}$ whereas signature 3 has its roots in homologous recombination deficiency during DNA double-strand break repair. ${ }^{20}$ The etiology of signature 12 has not yet been identified, although signature 26 is associated with a breakdown in DNA mismatch repair. The combination of signatures 3 and 26 may indicate, from an evolutionary perspective, how ALCL tumors accumulate mutations. Comparable patterns were found when comparing signatures to the COSMIC signature database ${ }^{22}$ (data not shown). There was no detectable difference between the mutational signature of pediatric $(n=4)$ or adult $(n=7) \mathrm{ALK}^{+}$ALCL patients (data not shown).

\section{Gene set enrichment analysis confirms the importance of T-cell receptor signaling, but also of the Notch pathway in $\mathrm{ALK}^{+}$anaplastic large cell lymphoma pathobiology}

Gene set enrichment analysis (GSEA) of mutated genes showed that TCR signaling and Notch pathways are enriched across all five databases used (Figure 2D; Online Supplementary Table S8). Further analysis of the domains frequently found in the mutated genes revealed an enrichment in proteins with epidermal growth factor (EGF)-like or calcium-ion binding domains (Figure 2E), two features of the NOTCH1 protein, and indeed the locus of both of the NOTCH1 mutations identified in this study (see below). Twenty of the 25 patient tumors carry mutations in proteins of the Notch pathway with a range of one to four and a median of two mutations per patient (Online Supplementary Table S8). Furthermore, reactome network clustering analysis ${ }^{23}$ showed TP53 as a key node, which is not unexpected as TP53 has been reported to play a key role in the pathogenesis of $\mathrm{ALCL}^{24}$ (Online Supplementary Figure S2E). Given the importance of the Notch pathway in T-cell biology, particularly in the developing thymus, which we proposed tp be the origin of $\mathrm{ALK}^{+} \mathrm{ALCL},{ }^{25}$ and the previous implication of the NOTCH1 pathway in the pathogenesis of $\mathrm{ALCL},{ }^{8}$ the NOTCH1 mutations detected and the NOTCH1 pathway were explored for their role in the pathogenesis of $\mathrm{ALK}{ }^{+}$ALCL. 


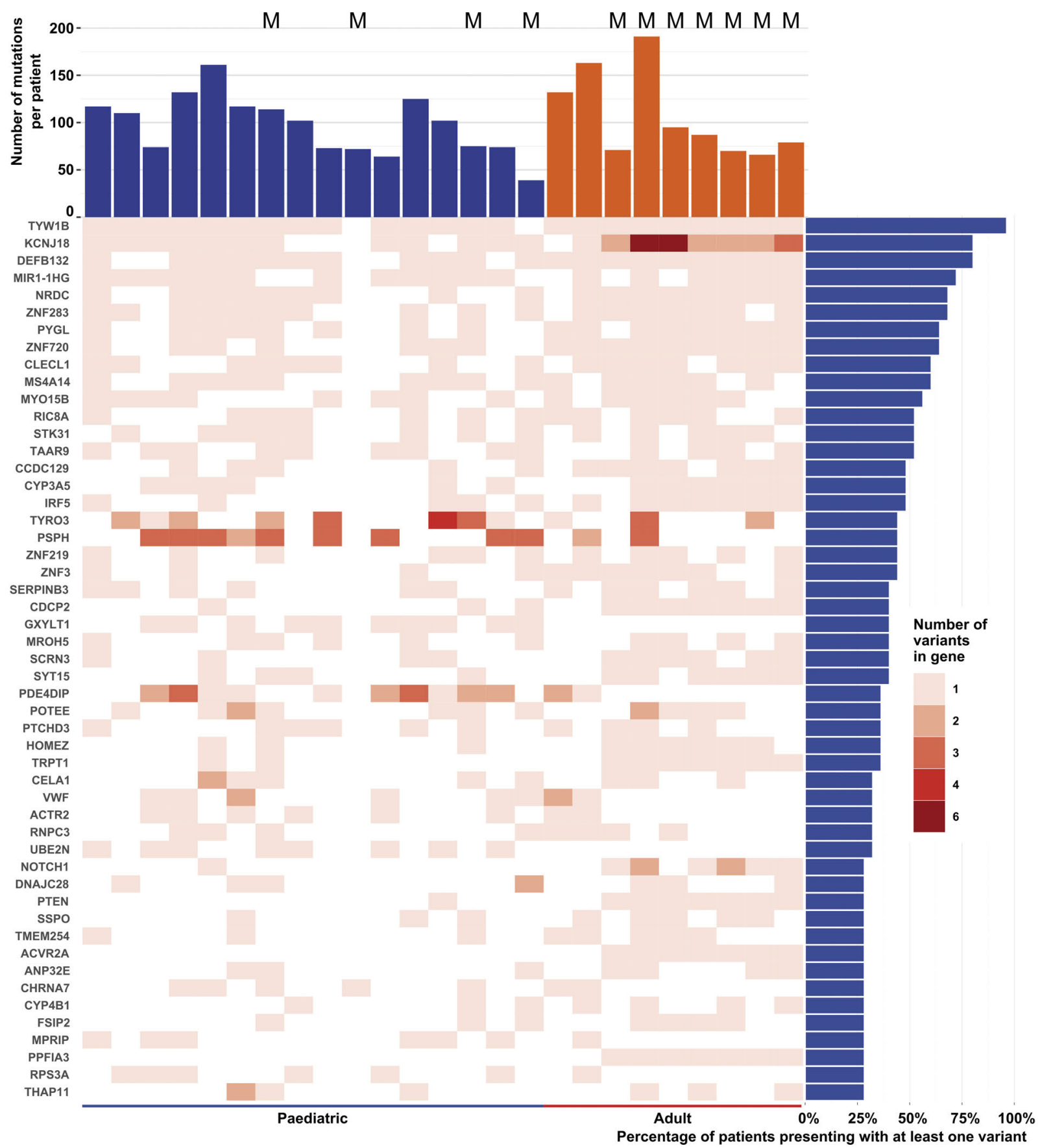

Figure 1. Summary of whole exome sequencing data. Individual results for each patient presented as a heatmap for genes found to be mutated in at least a quarter of patients. The number of different variants presenting in each gene is displayed for individual patients. The total number of variants identified for individual patients is also displayed above the graph. The panel on the right shows the percentage of patients presenting with at least one variant of the indicated gene. Pediatric and adult patients are separated. Patients for whom matched peripheral blood was sequenced are labeled with an ' $M$ '.

\section{NOTCH1 variant T349P provides a growth advantage to cells}

Of the $25 \mathrm{ALK}^{+}$ALCL tumor samples analyzed by WES, $24 \%$ presented with the NOTCH1 variant T349P, while $12 \%$ had the T311P variant. These data were validated by Sanger sequencing of a further 78 samples (including 18 of the samples previously analyzed by WES with a total of 55 ALK+ ALCL, and 23 ALK- ALCL) (Online Supplementary Table S2). In this validation cohort, the T349P variant was detected in $12 \%$ of patients $(n=78$;
$15 \%$ of $\mathrm{ALK}^{-}$patients and $9.3 \%$ of $\mathrm{ALK}^{+}$patients) (Online Supplementary Figure S3A) and the T311P variant was found in $7.6 \%$ of patients $\left(n=78 ; 10.2 \%\right.$ of $\mathrm{ALK}^{+}$patients, none in $\mathrm{ALK}^{-}$patients) (Online Supplementary Figure S3B). In the majority of cases, tumors presented with a mutation at either T311P or T349P and therefore the overall incidence of patients with at least one mutation of the EGF-like domain was $18 \%(n=78)$, although one patient presented with both mutations. We detected two additional NOTCH1 mutations, H1190P and G1503S, 
A
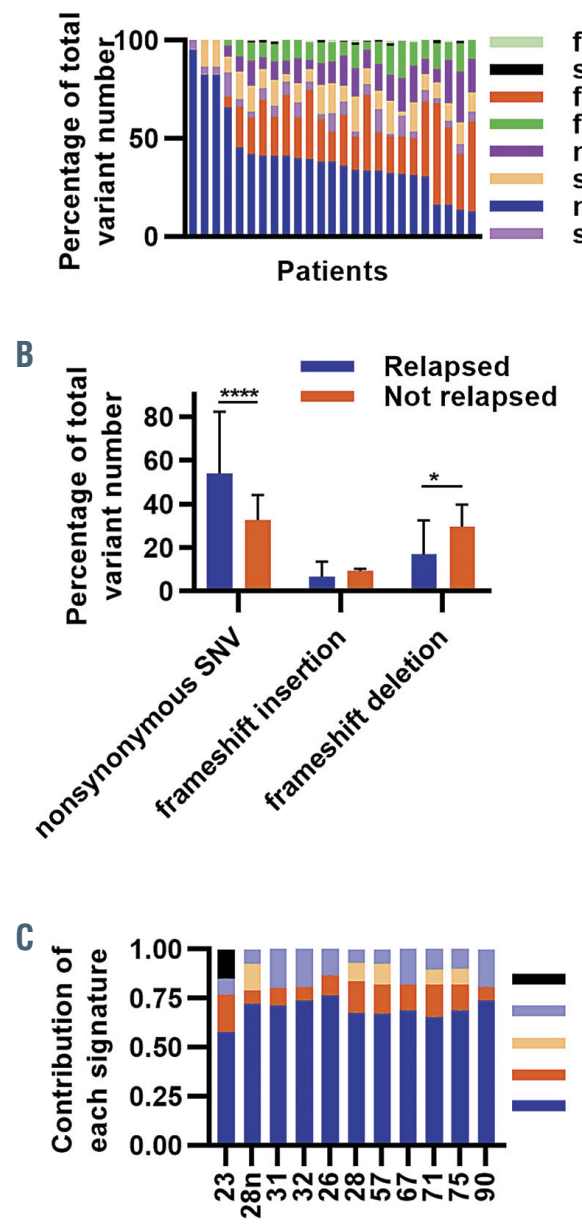

Patients
D

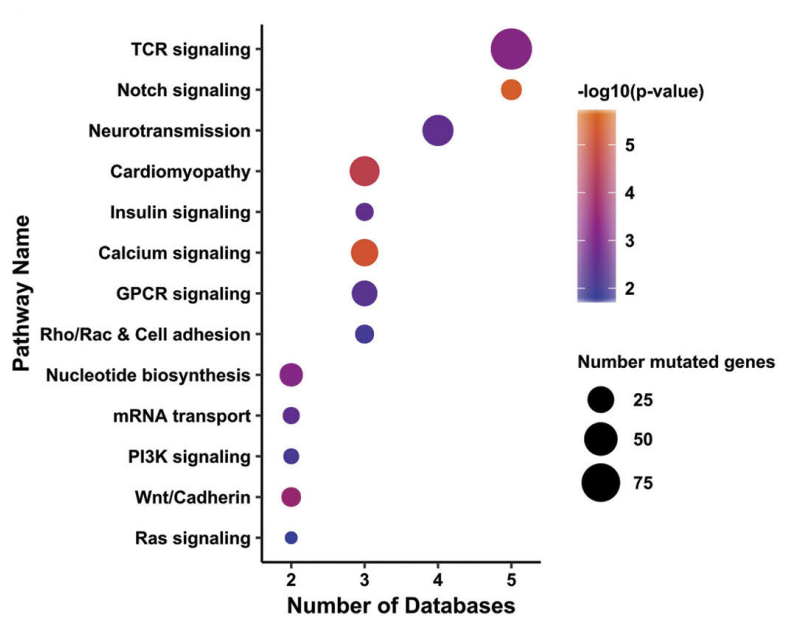

$\mathrm{E}$

Unknown signature Signature 26 Signature 12 Signature 3 Signature 1

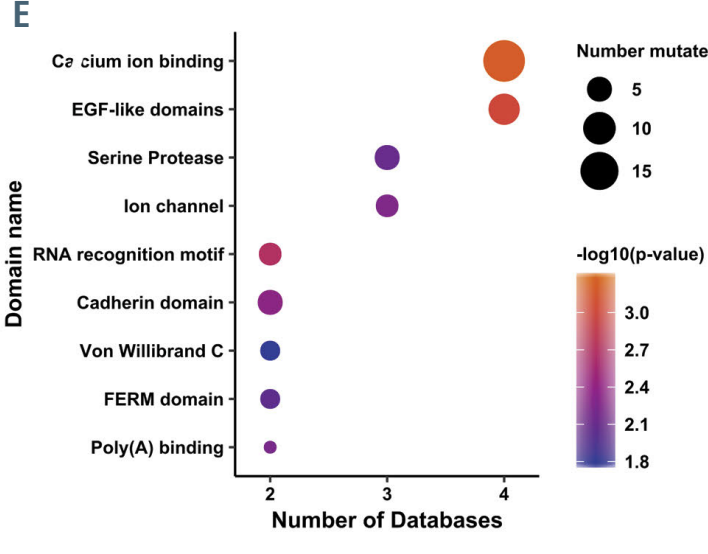

Figure 2. Analysis of whole exome sequencing data yields insights into the ALK+ anaplastic large cell lymphoma genomic landscape. (A) Proportion of variant type for each patient. (B) Mean and standard deviation of the proportion of variant types for patients who did ( $n=9)$ or did not ( $n=9)$ relapse within 5 years after diagnosis $(* * * P<0.001)$. (C) Mutational signatures ${ }^{20}$ derived from the variant profiles of each of the 11 patients for whom we hold sequenced matched peripheral blood. (D, E) Scatter plots of the pathways (D) or domains (E) found to be enriched in our dataset, displaying the number of databases in which each hit was found to be enriched, along with the $(-) \log _{10}$ of the statistical enrichment $P$-value of the software in which each hit was found to be most enriched, and the corresponding number of genes involved. SNV: single nucleotide variant.

although these were only detected in one patient each and so were not studied further. Of note, there was no significant difference in prognosis for patients presenting with NOTCH1 T349P and/or T311P over those with wild-type (WT) NOTCH1 when considering the whole cohort of patients (Online Supplementary Table S9, Online Supplementary Figure S3J). However, if adult patients were considered in isolation, there was a significant reduction in overall survival for those with NOTCH1 mutations $(P<0.05)$ but these data are based on just three patients and so should be interpreted with caution.

Variant T349P, at position 1045, is within the sixth of 34 exons of NOTCH1, which encodes one of the numerous EGF-like domains that make up the extracellular domain of NOTCH1 (EGF-like domain 9 of 36, which is a calcium-binding domain). NOTCH1 T349P was predicted to be a function-altering mutation by variant effect prediction software including SIFT (score $=0.01$ ) and PolyPhen (score $=0.999$ ), among others. ${ }^{26-30}$ Furthermore, the COSMIC database shows that T311 and T349 are the two most frequently reported mutated amino acids at the presumed NOTCH1/JAG1 interface across a range of cancers (including chronic myelomonocytic leukemia, ${ }^{31}$ chronic lymphocytic leukemia, ${ }^{32} \mathrm{~T}$-cell acute lymphoblastic leukemia, ${ }^{33}$ rhabdomyoscaroma ${ }^{34}$ and squamous cell carcinoma ${ }^{35}$ ) (Figure 3A).

To determine the impact of the detected NOTCH1 mutants on cell proliferation, WT, T349P or T311P mutants of NOTCH1 were expressed in HEK293FT cells (Figure $3 \mathrm{~B}$ and $\mathrm{C}$ ). These cells were chosen because of their low levels of endogenous NOTCH1 expression. In comparison, ALCL cell lines express high levels of WT NOTCH1. A significant increase in ATP production, suggestive of enhanced cell proliferation (as determined by a RealTime-Glo assay), was observed at $72 \mathrm{~h}$ for cells expressing the NOTCH1 T349P mutant as compared to WT NOTCH1 in the absence of exogenously applied ligand (Figure 3D). The T311P mutant also led to an increase in proliferation, although this was not statistically significant when compared to WT NOTCH1. However, a significant increase in proliferation was detected when using an MTT assay (Online Supplementary Figure S3I). In addition, the transcriptional targets of NOTCH1 activity, HES1 and HEY1, were expressed at higher levels in the 
A

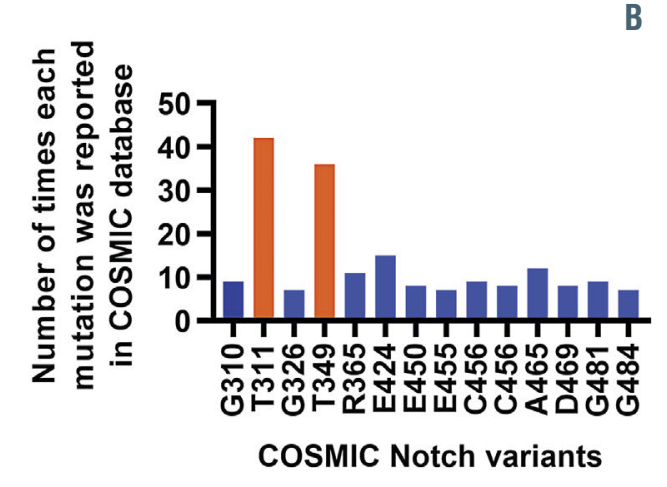

D

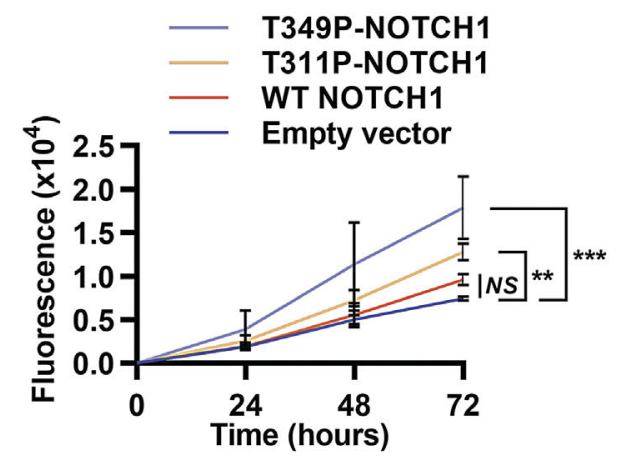

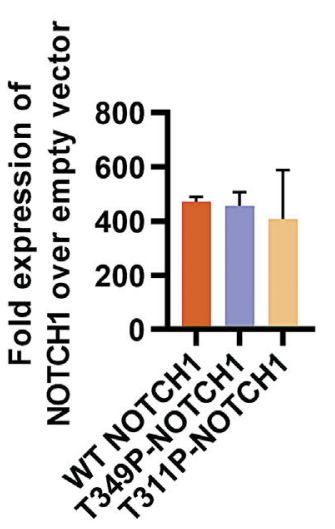

C

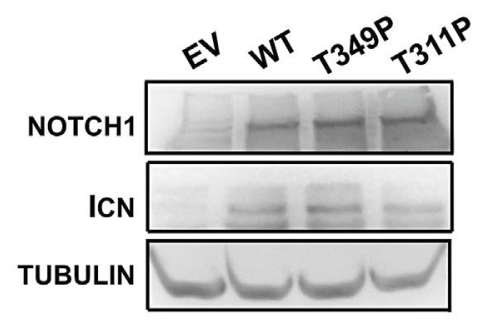

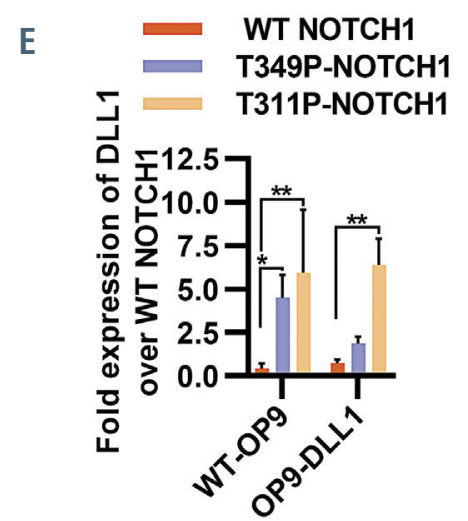

Figure 3. NOTCH1 T349P and T311P mutants confer a growth advantage to cells. (A) The most frequent variants (of the amino acids involved in the NOTCH1-JAG1 interface) of $\mathrm{NOTCH} 1$ reported on COSMIC, and the frequency at which they have been reported. (B) Quantitative polymerase chain reaction (qPCR) showing the foldincrease in transcript levels of NOTCH1 compared to that in cells transduced with an empty vector $(n=3)$. (C) Western blot for full-length and cleaved intracellular NOTCH1 (ICN) and $\alpha$-tubulin in HEK293FT cells $48 \mathrm{~h}$ after transfection to express wild-type (WT) or mutant (T349P or T311P) NOTCH1, or an empty vector (EV). Only the relevant sections of the whole blot are shown and the contrast of the whole image was modified in order to improve legibility. Data are representative of three biological repeats. (D) Proliferation of HEK293FT cells expressing the indicated proteins or empty vector control as measured by the Real Time Glo assay at 24,48 and $72 \mathrm{~h}$ after transfection ( $* * * P<0.001 ; \mathrm{n}=3$ ). (E) Fold-change in expression of DLL1 over the WT NOTCH1 control as assessed by qPCR in HEK293FT cells expressing the indicated proteins and co-cultured with either WT or DLL1-expressing OP9 cells $(* P<0.05 ; * * P<0.01 ; n=3)$. All bar plots display the mean of biological replicates, and error bars represent the standard deviation.

HEK293FT cells expressing NOTCH1 mutants compared to those transfected with either an empty vector or WT NOTCH1 (Online Supplementary Figure S3F and S3G). These data are suggestive of increased NOTCH1 activity as a consequence of the NOTCH1 T349P mutation compared to WT NOTCH1.

To determine why the T349P NOTCH1 mutant led to enhanced cell proliferation, in silico modeling was performed to predict the effects of the mutation on protein conformation using a published crystal structure of NOTCH1 bound to one of its ligands (Jagged1; PDBID 5UK5) (Online Supplementary Figure S3C and S3D). ${ }^{36}$ The model demonstrated that NOTCH1 residues 349 and 311 mediate binding to NOTCH1 ligands. Therefore, to determine whether increased cell proliferation (Figure 3D) was dependent on NOTCH1 ligand, NOTCH1 WT or mutant-expressing HEK293FT cells were co-cultured with OP9 cells expressing or not the NOTCH1 ligand DLL1. There was no discernible difference in proliferation between cells co-cultured with wild-type OP9, or OP9-DLL1 cells (Online Supplementary Figure S3E). However, increased transcription of endogenous DLL1 was observed on expression of the mutant forms of NOTCH1 compared to WT protein, suggesting that the mutant proteins might themselves lead to transcription of ligand in an autonomous fashion (Figure $3 \mathrm{E}$ ). In an attempt to validate this, we silenced DLL1 using a specific siRNA (Online Supplementary Figure S3H), which reduced the proliferation advantage induced by the NOTCH1 T349P mutant (Online Supplementary Figure S3I).

\section{NPM-ALK induces expression of NOTCH1 via STAT3 transcriptional activity in anaplastic large cell Iymphoma}

Given that ALCL cell lines express high levels of WT $\mathrm{NOTCH} 1,{ }^{8}$ the mechanism of NOTCH1 expression was investigated. NPM-ALK activity was inhibited by incubation with the ALK/ROS/MET inhibitor crizotinib (Figure 4A) or expression silenced with a specific shRNA (Figure 4D) in three and two cell lines, respectively. In both cases, a significant decrease in transcripts for NOTCH1 (Figure $4 \mathrm{~B}$ and $\mathrm{E}$ ) and its transcriptional target HES1 (Figure 4C and $\mathrm{F}$ ), was observed, suggesting that NOTCH1 transcription and NOTCH1 activity are dependent on NPM-ALK. Given that STAT3 is a key nodal downstream target of NPM-ALK, STAT3 expression was inhibited by employing specific shRNA in ALCL cell lines (Figure 4G). As predicted, transcript levels of NOTCH1 (Figure 4H), HES1 (Figure 4I) and HEY1 (Figure 4J) were all significantly downregulated as a result of STAT3 silencing in both $\mathrm{ALK}^{+}$and $\mathrm{ALK}^{-} \mathrm{ALCL}$ cell lines (Figure 4H-J, Online Supplementary Figure S4A). Analysis of published chro- 
A

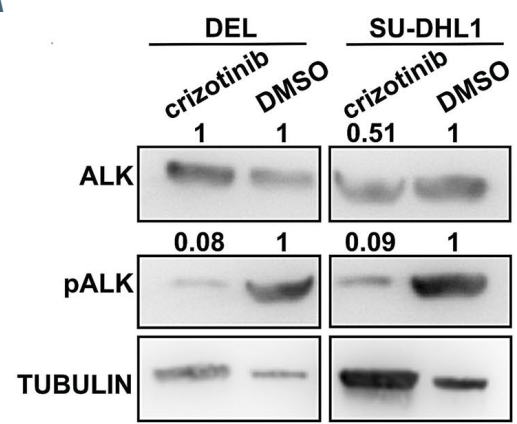

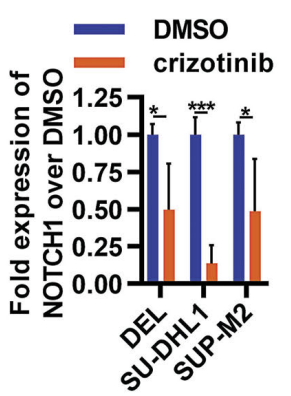

C

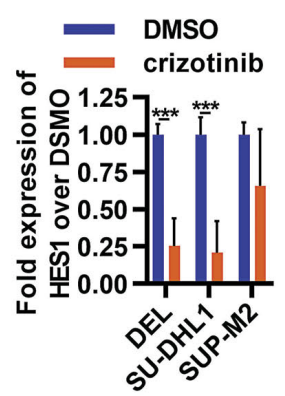

D

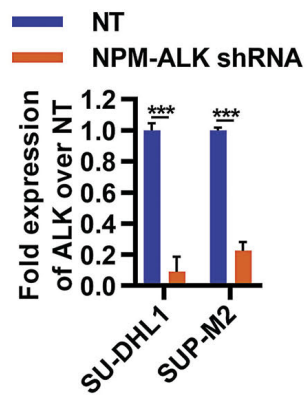

E

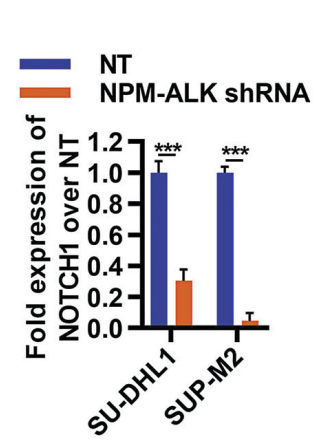

F

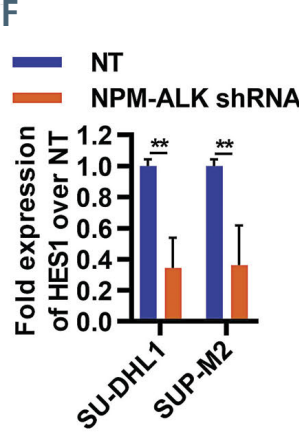

G
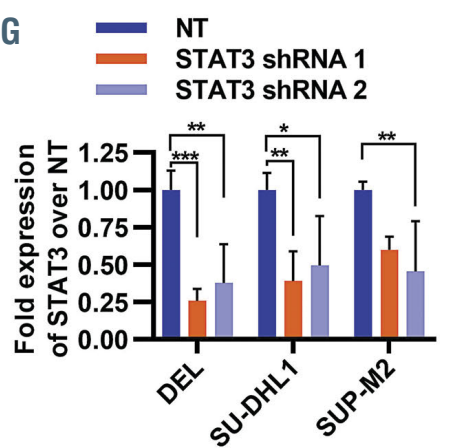

H
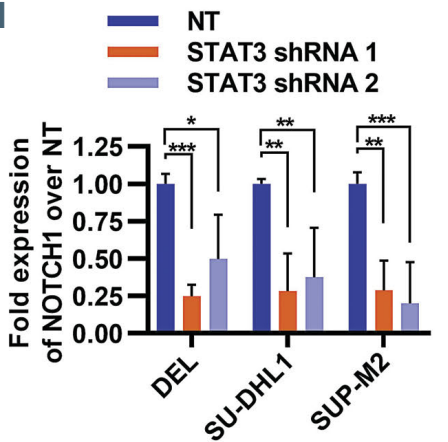

I
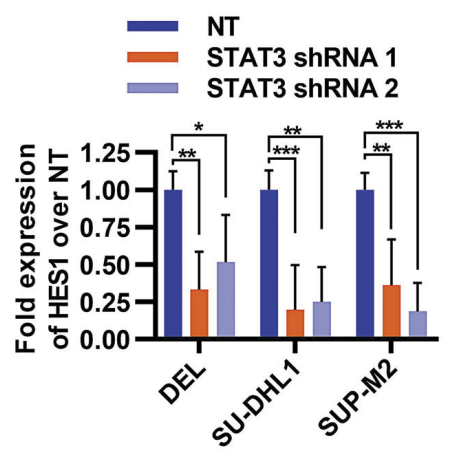

J
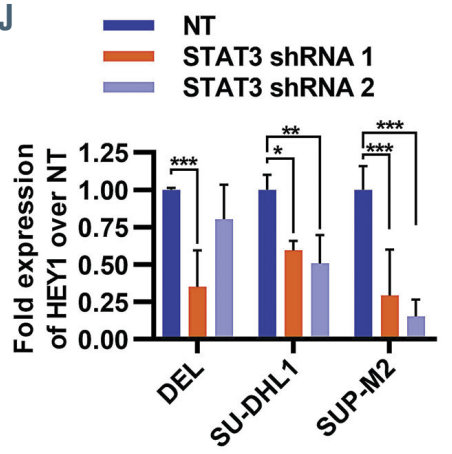

K

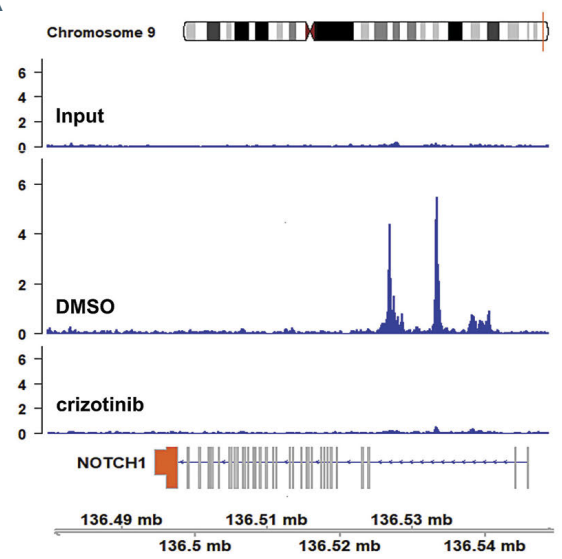

L

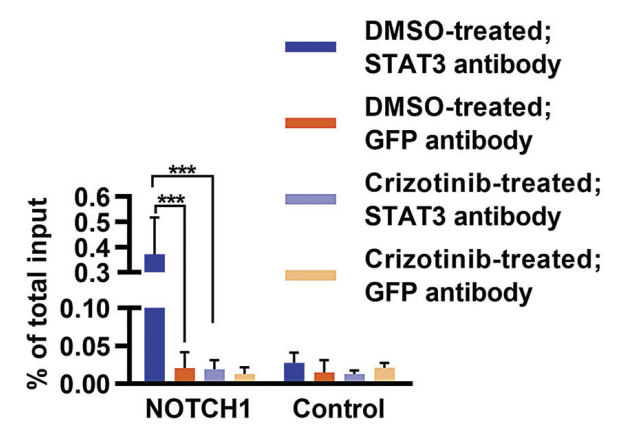

Figure 4. STAT3-mediated regulation of NOTCH1 by NPM-ALK in ALK ${ }^{+}$anaplastic large cell lymphoma. (A) Representative western blot for ALK, phospho-ALK and $\alpha$ tubulin in ALK+ anaplastic large cell lymphoma (ALCL) cell lines when treated with $300 \mathrm{nM}$ crizotinib or a vehicle control (dimethylsulfoxide, DMSO) for $6 \mathrm{~h}$. Only the relevant sections of the whole blot are shown and the contrast of the whole image was modified in order to to improve legibility. Data are representative of three biological replicates. Densitometry is included, as fold-change over the vehicle control and loading control. (B, C) Fold-change expression of NOTCH1 (B) and HES1 (C) over vehicle control in the indicated $\mathrm{ALK}^{+} \mathrm{ALCL}$ cell lines $48 \mathrm{~h}$ after treatment with crizotinib, as determined by quantitative polymerase chain reaction (qPCR) $(* P<0.05 ; * * P<0.01 ; * * * P<0.001 ; n=3)$. (D-F) Fold-change expression of NPM-ALK (D), NOTCH1 (E) and HES1 (F) over non-targeting control in ALK ${ }^{+}$ALCL cell lines $48 \mathrm{~h}$ after transduction with control non-targeting (NT) shRNA, or a shRNA targeting NPM-ALK, as determined by $\mathrm{qPCR}(* * P<0.01 ; * * * P<0.001$; $\mathrm{n}=3$ ). (G-J) Foldchange expression of STAT3 $(\mathrm{G})$, NOTCH1 (H), HES1 (I) and HEY1 (J) over non-targeting NT control in ALK ${ }^{+}$ALCL cell lines $48 \mathrm{~h}$ after transduction with control NT shRNA, or one of two shRNA targeting STAT3, as determined by qPCR ( $* * P<0.01 ; * * * P<0.001 ; n=3)$. (K) Binding of STAT3 to the promoter regions of NOTCH1 in SUDHL1 cells treated with a vehicle control (middle track) or crizotinib (lower track); the upper track is the input for two separate cell lines, data were obtained by analyzing previously published data. ${ }^{37}$ (L) Chromatin immunoprecipitation-qPCR binding of STAT3 and GFP at the NOTCH1 promoter region, or at a negative control intergenic region, in SUP-M2 cells treated with either a vehicle control or crizotinib ( $300 \mathrm{nM})$ for $6 \mathrm{~h}$, as determined by $\mathrm{qPCR}(* * * P<0.0001 ; \mathrm{n}=3$ ), expressed as the percentage of the total input. All measures of expression by qPCR were normalized to GAPDH and PPIA. All bar plots display the mean of biological replicates, and error bars represent standard deviations. 
A

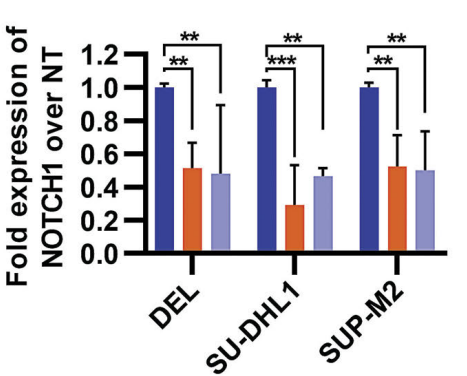

D

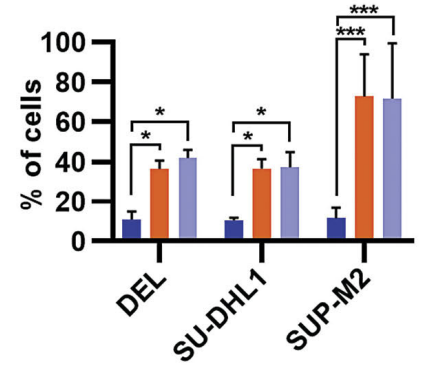

G

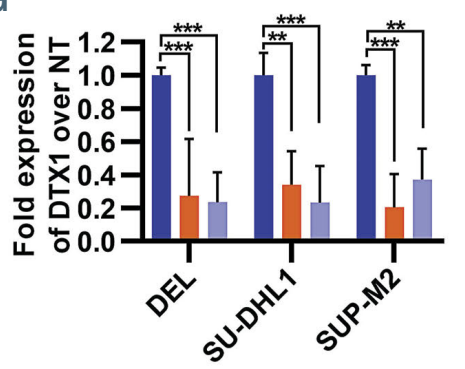

B

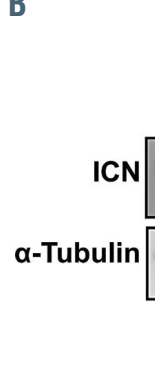

DEL

NT sh1 sh2 NT sh1 sh2

$\begin{array}{llllll}1 & 0.58 & 0.67 & 1 & 0.57 & 0.83\end{array}$

ICN
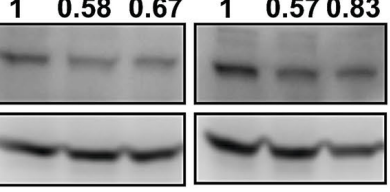

C

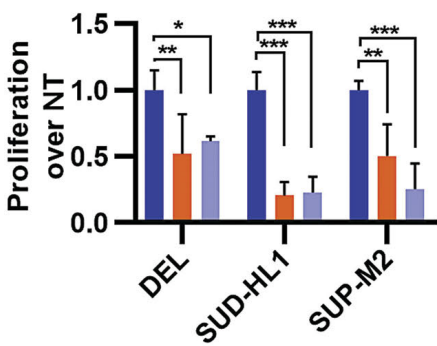

E

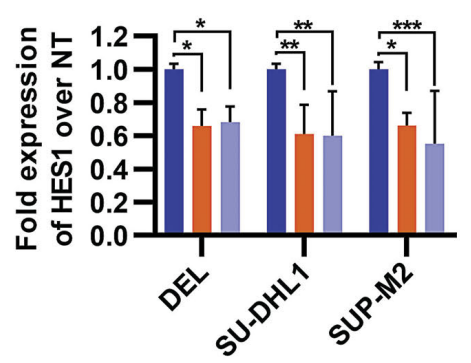

F

H

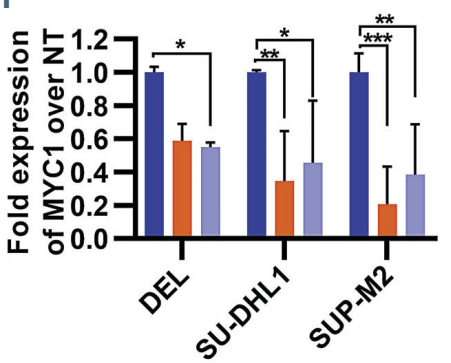

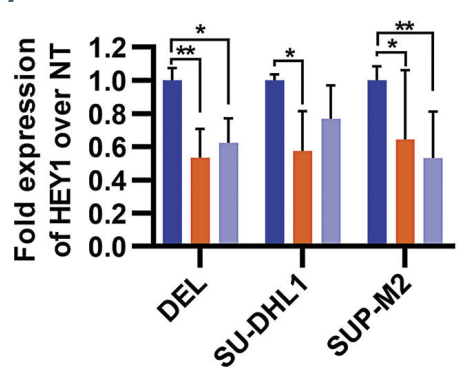

Non-targeting shRNA NOTCH1 shRNA-1 NOTCH1 ShRNA-2

Figure 5. Silencing NOTCH1 expression in anaplastic large cell lymphoma cell lines inhibits cell proliferation and induces cell death. (A) Fold change expression of NOTCH1 over non-targeting (NT) control (normalized to GAPDH and PPIA) in the indicated $\mathrm{ALK}^{+}$anaplastic large cell lymphoma (ALCL) cell lines $48 \mathrm{~h}$ after transduction with control non-targeting (NT) shRNA, or one of two shRNA targeting NOTCH1 as determined by quantitative polymerase chain reaction ( $* * P<0.01 ; * * * P<0.001$; $n=3$ ). (B) Representative western blot for cleaved intracellular NOTCH1 (ICN) and $\alpha$-tubulin in ALK ${ }^{+}$ALCL cell lines $48 \mathrm{~h}$ after transduction with NT control shRNA, or one of three shRNA targeting NOTCH1. Only the relevant sections of the whole blot are shown and the contrast of the whole image was modified in order to improve legibility. Data are representative of three biological repeats. Densitometry is included as fold-change over the vehicle control and loading control. (C) Proliferation of ALK ${ }^{+}$ALCL cell lines over the NT control shRNA, determined using an MTT assay $48 \mathrm{~h}$ after transduction with NT control shRNA, or one of two shRNA targeting NOTCH1 $(* P<0.05 ; * * P<0.01 ; * * * P<0.001 ; \mathrm{n}=3)$. (D) Quantification of the percentage of cells positive for annexin $\mathrm{V}$ and/or propidium iodide $48 \mathrm{~h}$ after transduction with NT control shRNA (left panel), or two shRNA targeting NOTCH1 (middle and right panels) $(* P<0.05 ; * * * P<0.001 ; n=3)$. (E-H) Fold-change of expression of HES1 (E), HEY1 (F), DTX1 (G) or MYC (H) over NT control shRNA transduced cells (normalized to GAPDH and PPIA) in the indicated ALK ${ }^{+}$ALCL cell lines $48 \mathrm{~h}$ after transduction with NT control shRNA, or one of two shRNA targeting NOTCH1, as determined by quantitative polymerase chain reaction ( $* P<0.05 ; * \star P<0.01 ; * \star \star P<0.001 ; n=3$ ). All bar plots display the mean of biological replicates, and error bars represent standard deviations; the bar plots are color-coded as indicated in the Figure.

matin immunoprecipitation sequencing data ${ }^{37}$ of STAT3 binding sites in the ALCL cell lines SU-DHL1 and JB6, treated with either crizotinib or a vehicle control, showed a significant decrease in binding of STAT3 at the NOTCH1 gene in crizotinib-treated cells (Figure 4K, Online Supplementary Figure S4B). These data were validated by chromatin immunoprecipitation-quantitative polymerase chain reaction analysis whereby a significant decrease in binding of STAT3 at the NOTCH1 gene upon crizotinib treatment in the SUP-M2 cell lines was observed (Figure 4L).

\section{NOTCH1 is a therapeutic target in $\mathrm{ALK}^{-}$and $\mathrm{ALK}^{+}$ anaplastic large cell lymphoma}

Given the expression and activity of NOTCH1 in ALCL, its utility as a therapeutic target was investigated. Expression of NOTCH1 was inhibited at both transcript
(Figure 5A, Online Supplementary Figure S5A) and protein levels (Figure $5 \mathrm{~B}$ ) by shRNA in both $\mathrm{ALK}^{+}$and $\mathrm{ALK}^{-} \mathrm{ALCL}$ cell lines leading to a significant decrease in cell proliferation at $48 \mathrm{~h}$, as measured by the MTT assay, in all four cell lines (Figure 5C, Online Supplementary Figure S5B) concomitant with a significant increase in the percentage of cells staining positive for either annexin- $V$ or propidium iodide, suggestive of cell death by apoptosis (Figure 5D). As expected, NOTCH1's transcriptional targets HES1 and HEY1 (Figures 5E and 5F, Online Supplementary Figure S5A) were also downregulated upon NOTCH1 silencing by shRNA. To further determine the signaling cascade downstream of NOTCH1, we made use of published microarray data examining the effect of GSI treatment on gene expression in T-cell acute lymphoblastic leukemia, ${ }^{38}$ ${ }^{40}$ and found genes such as MYC and DTX1 to be potential targets of NOTCH1 signaling (Online Supplementary Figure 

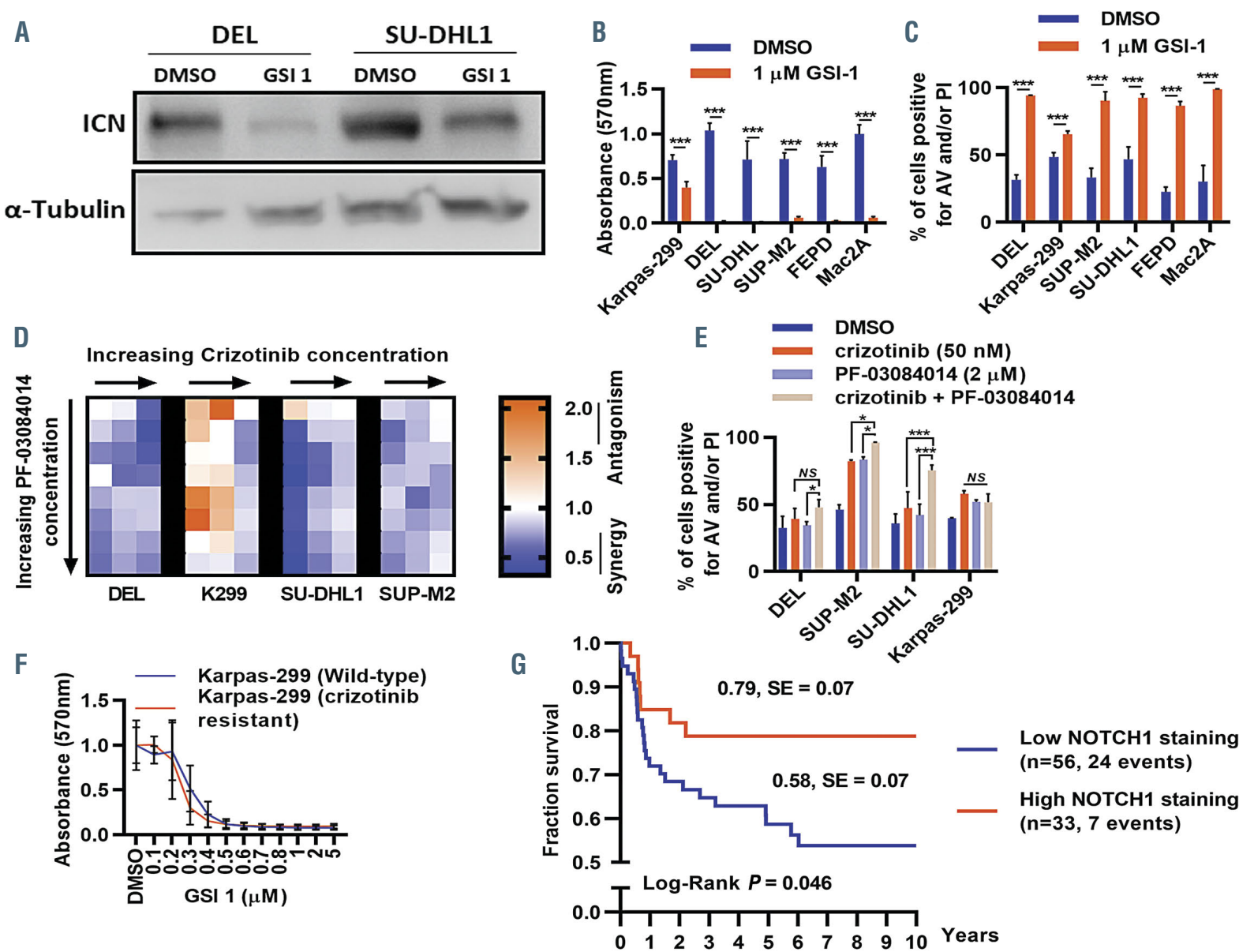

Low NOTCH1 staining ( $n=56,24$ events)

High NOTCH1 staining ( $n=33,7$ events)

Figure 6. ALK inhibitor sensitive and resistant anaplastic large cell lymphoma cell lines are sensitive to $\gamma$-secretase inhibitors. (A) Western blot for cleaved intracellular NOTCH1 (ICN) and $\alpha$-tubulin in $\mathrm{ALK}^{+}$anaplastic large cell lymphoma (ALCL) cell lines when treated with $1 \mu \mathrm{M} \mathrm{GSI-I} \mathrm{for} 48 \mathrm{~h}$. Only the relevant sections of the whole blot are shown and the contrast of the whole image was modified in order to improve legibility. Data are representative of three biological repeats. (B) Proliferation of a panel of $\mathrm{ALK}^{+}$and ALK $\mathrm{ALCL}$ cell lines treated with $1 \mu \mathrm{M}$ of GSI-I for $48 \mathrm{~h}$, compared to vehicle control, as measured by the MTT assay $(* * * P<0.0001 ; \mathrm{n}=3)$. (C) Quantification of the percentage of cells positive for annexin $\mathrm{V}(\mathrm{AV})$ and/or propidium iodide (PI) when treated with either vehicle control or $1 \mathrm{uM}$ GSI-I for $48 \mathrm{~h}(* * * P<0.001 ; \mathrm{n}=3)$. (D) BLISS matrix showing the combination index on treating the indicated ALK ${ }^{+} \mathrm{ALCL}$ cell lines with crizotinib and PF03084014 for $72 \mathrm{~h}$ (using a range of concentrations from 25 to $100 \mathrm{nM}$ for crizotinib, and from $100 \mathrm{nM}$ to $10 \mu \mathrm{M}$ for PF-03084014). A combination index of <1 indicates synergy between drugs, 1 indicates additive effects, $>1$ indicates antagonistic effects $(n=3)$. (E) Quantification of the percentage of cells positive for AV and/or PI when treated with either vehicle control, 50 nM crizotinib, $2 \mu \mathrm{M}$ PF-03084014 or a combination of PF-03084014 and crizotinib for $48 \mathrm{~h}$ (NS: not significant: $* P<0.05 ; * * * P<0.00 ; n=3$ ). (F) Proliferation over vehicle control of wild-type or crizotinib-resistant Karpas-299 cells when treated with increasing concentrations of GSI-I, as measured by the MTT assay $(n=3)$. (G) Ten-year event-free survival of patients with ALK ${ }^{+}$anaplastic large cell lymphoma showing either little or no ( $n=56$ ), or strong $(n=33)$ NOTCH1 expression. All bar plots display the mean of biological replicates and error bars represent standard deviations.

S5C). In keeping with these data, we show that silencing NOTCH1 by shRNA in the ALK ${ }^{+}$ALCL cell lines DEL, SU-DHL1 and SUP-M2 (and the ALK- ALCL cell line FEPD) leads to significant decreases in both MYC and DTX1 transcript levels (Figure 5G and $\mathrm{H}$, Online Supplementary Figure $S 5 A$ ) and protein levels (Online Supplementary Figure S5D), suggesting that NOTCH1 in ALCL signals through a number of pathways beyond HES1 and HEY1. Indeed, analysis of published microarray data suggests that the expression of $\mathrm{MYC}$ and NOTCH1, and DTX1 and NOTCH1 correlates in both $\mathrm{ALK}^{+}$and $\mathrm{ALK}^{-} \mathrm{ALCL}$, but not in reactive lymph nodes (Online Supplementary Figure S5E-J).

Given the reduced cell proliferation and increased cell death observed on shRNA-mediated knockdown of NOTCH1 expression, ALCL cell lines were incubated with two different GSI: GSI-I (Z-LLNle-CHO) and PF$03084014 .{ }^{41}$ GSI-I inhibited NOTCH1 cleavage, as shown by a decrease in expression of the intracellular domain of
NOTCH1 (ICN) $48 \mathrm{~h}$ after drug treatment (Figure 6A), concomitant with a decrease in cell proliferation, as shown by the MTT assay (Figure 6B), and an increase in apoptosis, as determined by positive cell surface staining for annexin $\mathrm{V}$ and/or intracellular propidium iodide (Figure 6C, Online Supplementary Figure S6A). In contrast, PF-03084014 did not significantly affect cell proliferation or apoptosis on its own at any of the concentrations assessed, ranging from $10 \mathrm{nM}$ to $10 \mu \mathrm{M}$ for up to $72 \mathrm{~h}$ of incubation (data not shown). None of the ALCL cell lines used in the research described here carries either the NOTCH1 T311P or T349P mutation.

\section{Gamma secretase inhibitors synergize with ALK inhibitors to induce cell death}

Co-incubation of three of four ALCL cell lines with either PF-03084014 or GSI-1 with crizotinib led to additive to synergistic activity in reducing cell proliferation, as indicated by a Bliss Independence Index of less than one 
across several concentrations (Figure 6D, Online Supplementary Figure S6B). Karpas 299 cells have different genetic defects, which may explain the antagonistic results in this cell line. ${ }^{42}$ Indeed, a significant increase in apoptotic cells was observed following $48 \mathrm{~h}$ of treatment with a combination of $50 \mathrm{nM}$ crizotinib and $2 \mu \mathrm{M}$ PF03084014 (Figure 6E, Online Supplementary Figure S6C).

Ideally, a single-agent ALK inhibitor would provide a less toxic frontline treatment approach in the future, although resistance would be expected to develop. Therefore, crizotinib-resistant ALCL cell lines were assessed for their sensitivity to GSI which led to a significant decrease in cell proliferation (Figure 6F, Online Supplementary Figure S6D-F). These data suggest the potential use of GSI as either second-line treatment for ALK inhibitor-resistant disease, or frontline therapy in combination with ALK tyrosine kinase inhibitors.

\section{NOTCH1 is a potential biomarker predictive of relapse in $\mathrm{ALK}^{+}$anaplastic large cell lymphoma}

A clinically annotated FFPE tissue microarray of $89 \mathrm{ALK}^{+}$ ALCL patient samples, biopsied at the time of the patients' initial presentation, was analyzed for cleaved NOTCH1 protein expression (Online Supplementary Figure S6G). Of the 89 patient tumors assessed, $88.8 \%$ showed high cleaved NOTCH1 staining (moderate and strong staining categories), in keeping with previously published results. ${ }^{7,8,43}$ Interestingly, patients with low cleaved NOTCH1 staining (negative and weak staining categories) are more likely to relapse (based on the 10-year event-free survival) and have a significantly worse prognosis $(P<0.05)$ (Figure 6G).

\section{Discussion}

The genetics underlying $\mathrm{ALK}^{+} \mathrm{ALCL}$ at the level of somatic mutations remains largely unknown. Being a relatively rare cancer with a common, well-characterized driving oncogenic event, more could be done to uncover other genetic alterations. We therefore sequenced $18 \mathrm{ALK}^{+} \mathrm{ALCL}$ tumors and analyzed their genome together with seven previously reported $\mathrm{ALK}^{+}$cases $^{9}$ in order to uncover pathogenic mechanisms and novel therapeutic targets. Mutational signature analysis showed signatures 1,3 and 26 in all our patient samples. The latter two signatures have their roots in homologous recombination DNA double-strand-break repair and mismatch DNA repair deficien$\mathrm{cy}^{20}$ This suggests that DNA damage repair mechanisms might be impaired in these patients, predisposing them to ALCL, perhaps through germline mutations in DNA repair proteins (such as a BRCA2 variant K3326X, COSM4984873, found in germline sample $67 \mathrm{~B}^{44}$ ). In contrast, signature 1 , accounting for the majority of the total contribution, is associated with a prevalence of $\mathrm{C}>\mathrm{T}$ transitions at NpCpG trinucleotides and spontaneous deamination of 5-methyl-cytosine, considered an age-related phenomenon due to endogenous mutational processes. ${ }^{21}$ In general, 5-methyl cytosine residues are unstable within DNA and are prone to mutation, representing hotspots for this activity. ${ }^{45}$ This is paradoxical with the young age range of $\mathrm{ALK}^{+}$ALCL patients, with the majority being young adults, and suggests that the mechanism(s) leading to such mutations may be 'speeded up'. Indeed, CpG transitions may be a consequence of secondary factors that promote deamination, such as exogenous mutagens and carcinogens, for example polycyclic aromatic hydrocarbons. ${ }^{45}$
To identify pathways that are key to ALCL biology, GSEA was employed and revealed a number of pathways commonly affected by mutations in $\mathrm{ALK}^{+}$ALCL. Interestingly, the TCR signaling pathway was prominent in our analysis. It has previously been shown that NPMALK can substitute for key TCR-induced distal signaling pathways and silencing of proximal proteins has been shown in ALCL. ${ }^{46}$ Another key pathway identified was NOTCH1; gain-of-function mutations in NOTCH1 have previously been identified in a number of other cancers, most notably in approximately 50 to $60 \%$ of T-cell acute lymphoblastic leukemia. ${ }^{47,48}$ However, most of these mutations are in the intracellular domains of the protein, with few reported in the extracellular domains. ${ }^{49-51}$ In contrast, novel mutations in the EGF-like domain of extracellular NOTCH1 were detected in $9.3 \%$ (T349P) and 10.2\% (T311P) of $\mathrm{ALK}^{+}$ALCL patients analyzed in this study. Predicted to be deleterious, the functional significance of these mutations was investigated using bioinformatics analysis. It has previously been shown that EGF-like domains 8 to 12 are important for NOTCH1 binding to its ligands. ${ }^{36,52}$ Specifically, threonine bases, lost in T349P and T311P, within the EGF-like domains are post-translationally modified by O-linked glycosylation which is necessary for ligand-engaged NOTCH1 signaling. . $^{53-55}$ Mutations to proline, a rigid and bulky amino acid, result in a change in tertiary structure, often forcing a change in $\beta$-sheet conformation (EGF-like domains are made of $\beta$-sheets among others). We demonstrated the positive impact of the T349P mutant on NOTCH1 activity, as shown by enhanced cell proliferation when expressed in HEK293 cells. We theorize that NOTCH1 T349P could modulate ligand binding (either directly or through modulation of calcium binding, particularly as calcium signaling is thought to be dysregulated in ALCL and calcium ions play an important role in NOTCH1 ligand binding ${ }^{56,57}$ ).

Regardless of the presence of NOTCH1 mutations, NOTCH1 constitutes a therapeutic target in ALCL independently of ALK status; suppression of NOTCH1 expression or activity led to an increase in apoptosis, in keeping with previous reports. ${ }^{7,8,58}$ Intriguingly, NPM-ALK has previously been shown to be sufficient to induce NOTCH1 expression. ${ }^{25}$ Not only did we confirm this by silencing NPM-ALK in ALCL, but we also showed that NPM-ALK acts through STAT3, which binds to the NOTCH1 promoter. This could explain why we observed synergistic effects between crizotinib and GSI in inducing cell death despite our evidence that NPM-ALK promotes NOTCH1 expression. Indeed, studies have shown that crizotinib synergizes with brentuximab vedotin, which targets CD30-expressing cells, despite NPM-ALK having been shown to drive CD30 expression. ${ }^{59}$ ALK inhibitors are now being added to frontline therapy ${ }^{60}$ (e.g., in trial NCT01979536) although this combination has led to some unexpected toxicity. ${ }^{11}$ NOTCH1 inhibition may therefore serve as a second-line treatment. Indeed, although GSI have suffered from gastrointestinal-related toxicity, isoform-specific GSI or antibody-based treatments that target NOTCH1 directly (NCT03422679) have shown more promise. ${ }^{61}$ Published studies variously describe that GSI-1 (both as a single treatment and in combination) and PF-03084014 are relatively well tolerated. ${ }^{62-64}$

Ultimately, our data show that GSI and ALK inhibitors act additively/synergistically and induce apoptosis of ALCL cell lines, and furthermore that ALK inhibitor-resis- 
tant cell lines remain sensitive to NOTCH1 inhibition. Hence, inhibition of NOTCH1 via GSI might represent a therapeutic option for both treatment-naive and ALK inhibitor-resistant ALCL - while NOTCH1 expression may be a viable biomarker predictive of relapse.

\section{Disclosures}

No conflicts of interest to disclose.

\section{Contributions}

HL: conceptualization, methodology, investigations, writing of the original draft, and visualization. NP: conceptualization, investigations, writing, review and editing. JDM: software, visualization, formal analysis, writing, review and editing. MS, SH, EN, MFM, AE, MC, LM, CGP, GH, CL, SP, AJ, AF, $A M, O W, G I, L L, O M, W K, S M, I A, M W, G A A B$ and $L K$ : resources. AFA: investigations, software. SPD: investigation, writing, review and editing. $W W$ and $C D W$ : resources, writing, review and editing. MZ: software and formal analysis. TLB: conceptualization, methodology, software, writing, review and editing. SDT: conceptualization, resources, writing, review, editing, supervision, project administration, and acquisition of funding.

\section{Acknowledgments}

The authors would like to thank Professor Christopher Aster and Dr Johnson (Harvard Medical School) for providing the fulllength NOTCH1 cDNA. We also thank: the Cambridge NIHR BRC Cell Phenotyping Hub; Medical Research Laboratories Core for Sanger sequencing; Washington State University Genomic Sequencing for library preparation, quality control and sequencing; the CCLG Tissue Bank, the CCLG centers and the ECMC Pediatric Network for the collection and provision of tissue samples. We especially thank the patients and families who voluntarily donated the samples.

\section{Funding}

This work was supported by grants from the Ministry of Science, Kingdom of Saudi Arabia to SDT, AI and SM (grant number 74497) and Bloodwise to SDT (grant number 12065). HL is supported by a Department of Pathology, University of Cambridge Pathology Centenary Fund PhD studentship. $S D T, L K, O M, S K, N P, S P D, C G P, W W, C D W$ and $C L$ are recipients of funds from a European Union Horizon 2020 Marie Sklodowska-Curie Innovative Training Network (ITNETN) grant, award n. 675712. CL is supported by a Czech Science Foundation research grant n. 19-23424Y and by research infrastructures EATRIS-CZ (LM2015064) and the NCMG (LM2015091) funded by MEYS CR. WK is supported by the KinderkrebsInitiative Buchholz, Holm-Seppensen. The CCLG Tissue Bank is funded by Cancer Research UK and CCLG.

\section{References}

1. Alessandri AJ, Pritchard SL, Schultz KR, Massing BG. A population-based study of pediatric anaplastic large cell lymphoma. Cancer. 2002;94(6):1830-1835.

2.Lamant L, Dastugue N, Pulford K, et al. A new fusion gene TPM3-ALK in anaplastic large cell lymphoma created by a (1;2)(q25;p23) translocation. Blood. 1999;93 (9):3088-3095

3. Morris SW, Kirstein MN, Valentine MB, et al. Fusion of a kinase gene, ALK, to a nucleolar protein gene, NPM, in non-Hodgkin's lymphoma. Science. 1994;263(5151):12811284.

4. Youssif C, Goldenbogen J, Hamoudi R, et al. Genomic profiling of pediatric ALK-positive anaplastic large cell lymphoma: a Children's Cancer and Leukaemia Group study. Genes Chromosom. Cancer. 2009;48(11):1018-1026.

5. Salaverria I, Beà S, Lopez-Guillermo A, et al. Genomic profiling reveals different genetic aberrations in systemic ALK-positive and ALK-negative anaplastic large cell lymphomas. Br J Haematol. 2008;140(5): 516-526.

6.Boi M, Rinaldi A, Kwee I, et al. PRDM1/BLIMP1 is commonly inactivated in anaplastic large T-cell lymphoma. Blood. 2013;122(15):2683-2693.

7.Kamstrup MRR, Biskup E, Gjerdrum LMR, et al. The importance of Notch signaling in peripheral T-cell lymphomas. Leuk Lymphoma. 2014:55(3):639-644.

8.Jundt F, Anagnostopoulos I, Förster R, et al. Activated Notch1 signaling promotes tumor cell proliferation and survival in Hodgkin and anaplastic large cell lymphoma. Blood. 2002;99(9):3398-3403.

9. Crescenzo R, Abate F, Lasorsa E, et al. Convergent mutations and kinase fusions lead to oncogenic STAT3 activation in anaplastic large cell lymphoma. Cancer Cell. 2015;27(4):516-532

10. Brugières L, Pacquement H, Le Deley M-C, et al. Single-drug vinblastine as salvage treatment for refractory or relapsed anaplastic large-cell lymphoma: a report from the French Society of Pediatric Oncology. J Clin Oncol. 2009;27(30):50565061.

11. Prokoph N, Larose H, Lim MS, Burke GAA, Turner SD. Treatment options for paediatric anaplastic large cell lymphoma (ALCL): current standard and beyond. Cancers (Basel). 2018;10(4):99

12. Minard-Colin V, Brugières L, Reiter A, et al. Non-Hodgkin lymphoma in children and adolescents: progress through effective collaboration, current knowledge, and challenges ahead. J Clin Oncol. 2015;33(27): 2963-2974.

13.Le Deley M-C, Rosolen A, Williams DM, et al. Vinblastine in children and adolescents with high-risk anaplastic large-cell lymphoma: results of the randomized ALCL99vinblastine trial. J Clin Oncol. 2010;28(25): 3987-3993

14. Gritti G, Boschini C, Rossi A, et al. Primary treatment response rather than front line stem cell transplantation is crucial for long term outcome of peripheral T-cell lymphomas. PLoS One. 2015;10(3):e0121822.

15. Gambacorti-Passerini C, Mussolin L, Brugieres L. Abrupt relapse of ALK-positive lymphoma after discontinuation of crizotinib. N Engl J Med. 2016;374(1):95-96.

16. Gambacorti-Passerini C, Messa C, Pogliani EM. Crizotinib in anaplastic large-cell lymphoma. N Engl J Med. 2011;364(8):775-776.

17. Mossé YP, Voss SD, Lim MS, et al. Targeting ALK with crizotinib in pediatric anaplastic large cell lymphoma and inflammatory myofibroblastic tumor: a Children's Oncology Group study. J Clin Oncol. 2017;35(28):3215-3221

18. Abramov D, Oschlies I, Zimmermann M, et al. Expression of CD8 is associated with non-common type morphology and outcome in pediatric anaplastic lymphoma kinase-positive anaplastic large cell lymphoma. Haematologica. 2013;98(10):15471553

19. Ait-Tahar K, Damm-Welk C, Burkhardt B, et al. Correlation of the autoantibody response to the ALK oncoantigen in pediatric anaplastic lymphoma kinase-positive anaplastic large cell lymphoma with tumor dissemination and relapse risk. Blood. 2010;115(16):3314-3319.

20. Alexandrov LB, Nik-Zainal S, Wedge DC, et al. Signatures of mutational processes in human cancer. Nature. 2013;500(7463):415421

21.Helleday T, Eshtad S, Nik-Zainal S. Mechanisms underlying mutational signatures in human cancers. Nat Rev Genet. 2014;15(9):585-598.

22.https://cancer.sanger.ac.uk/cosmic/signatures.

23. Fabregat A, Jupe S, Matthews L, et al. The reactome pathway knowledge base. Nucleic Acids Res. 2018;46(D1):D649 D655

24. Cui Y-X, Kerby A, McDuff FKE, Ye $\mathrm{H}$, Turner SD. NPM-ALK inhibits the p53 tumor suppressor pathway in an MDM2 and JNK-dependent manner. Blood. 2009;113(21):5217-5227.

25. Malcolm TIMM, Villarese P, Fairbairn CJ, et al. Anaplastic large cell lymphoma arises in thymocytes and requires transient TCR expression for thymic egress. Nat Commun. 2016;7:10087.

26. Adzhubei IA, Schmidt S, Peshkin L, et al. A method and server for predicting damaging missense mutations. Nat Methods. 2010;7 (4):248-249

27. Kumar P, Henikoff S, Ng PC. Predicting the effects of coding non-synonymous variants on protein function using the SIFT algorithm. Nat Protoc. 2009;4(7):1073-1082 
28. Chun S, Fay JC. Identification of deleterious mutations within three human genomes. Genome Res. 2009;19(9):1553-1561.

29. Schwarz JM, Cooper DN, Schuelke M, Seelow D. Mutationtaster2: mutation prediction for the deep-sequencing age. Nat Methods. 2014;11(4):361-362.

30. Choi Y, Sims GE, Murphy S, Miller JR, Chan AP. Predicting the functional effect of amino acid substitutions and indels. PLoS One. 2012;7(10):e46688.

31. Mason CC, Khorashad JS, Tantravahi SK, et al. Age-related mutations and chronic myelomonocytic leukemia. Leukemia. 2016;30(4):906-913.

32.Ljungström V, Cortese D, Young E, et al. Whole-exome sequencing in relapsing chronic lymphocytic leukemia: clinical impact of recurrent RPS15 mutations. Blood. 2016;127(8):1007-1016.

33. Neumann M, Vosberg S, Schlee C, et al. Mutational spectrum of adult T-ALL. Oncotarget. 2015;6(5):2754-2766.

34. Kohsaka S, Shukla N, Ameur N, et al. A recurrent neomorphic mutation in MYOD1 defines a clinically aggressive subset of embryonal rhabdomyosarcoma associated with PI3K-AKT pathway mutations. Nat Genet. 2014;46(6):595-600

35. Martin D, Abba MC, Molinolo AA, et al. The head and neck cancer cell oncogenome: a platform for the development of precision molecular therapies. Oncotarget. 2014;5(19):8906-8923.

36.Luca VC, Kim BC, Ge C, et al. NotchJagged complex structure implicates a catch bond in tuning ligand sensitivity. Science. 2017;355(6331):1320-1324.

37. Menotti M, Ambrogio C, Cheong T-C, et al. Wiskott-Aldrich syndrome protein (WASP) is a tumor suppressor in T cell lymphoma. Nat Med. 2019;25(1):130-140.

38. Choi SH, Severson E, Pear WS, et al. The common oncogenomic program of NOTCH1 and NOTCH3 signaling in T-cell acute lymphoblastic leukemia. PLoS One. 2017;12(10):e0185762.

39. Sanchez-Martin M, Ambesi-Impiombato A, Qin Y, et al. Synergistic antileukemic therapies in NOTCH1-induced T-ALL. Proc Natl Acad Sci U S A. 2017;114(8):2006-2011.

40. Wang H, Zou J, Zhao B, et al. Genomewide analysis reveals conserved and divergent features of Notch1/RBPJ binding in human and murine T-lymphoblastic leukemia cells. Proc Natl Acad Sci U S A. 2011;108(36):14908-14913

41.Locatelli MA Aftimos P, Dees EC, et al. Phase I study of the gamma secretase inhibitor PF-03084014 in combination with docetaxel in patients with advanced triplenegative breast cancer. Oncotarget. 2017;8(2):2320-2328.

42. Turturro F, Frist AY, Arnold MD, Seth $P$, Pulford K. Biochemical differences between SUDHL-1 and KARPAS 299 cells derived from $\mathrm{t}(2 ; 5)$-positive anaplastic large cell lymphoma are responsible for the different sensitivity to the antiproliferative effect of p27(Kip1). Oncogene. 2001;20(33):44664475.

43. Kamstrup MR, Ralfkiaer E, Skovgaard GL, Gniadecki R. Potential involvement of Notch1 signalling in the pathogenesis of primary cutaneous CD30-positive lymphoproliferative disorders. Br. J. Dermatol. 2008;158(4):747-753.

44. Meeks HD, Song H, Michailidou K, et al. BRCA2 polymorphic stop codon K3326X and the risk of breast, prostate, and ovarian cancers. J Natl Cancer Inst. 2015;108(2) div315.

45.Pfeifer GP. Mutagenesis at methylated CpG sequences. Curr Top Microbiol Immunol 2006;301:259-281.

46. Turner SD, Yeung D, Hadfield K, Cook SJ, Alexander DR. The NPM-ALK tyrosine kinase mimics TCR signalling pathways, inducing NFAT and AP-1 by RAS-dependent mechanisms. Cell Signal. 2007;19(4):740-747.

47. Aster JC, Blacklow SC, Pear WS. Notch signalling in T-cell lymphoblastic leukaemia/lymphoma and other haematological malignancies. J Pathol. 2011;223(2): 262-273.

48. Breit S, Stanulla M, Flohr T, et al. Activating NOTCH1 mutations predict favorable early treatment response and long-term outcome in childhood precursor T-cell lymphoblastic leukemia. Blood. 2006;108(4):1151-1157.

49. Wang NJ, Sanborn Z, Arnett KL, et al. Lossof-function mutations in Notch receptors in cutaneous and lung squamous cell carcinoma. Proc Natl Acad Sci U S A. 2011;108(43):17761-17766.

50. Vollbrecht C, Mairinger FD, Koitzsch U, et al. Comprehensive analysis of disease-related genes in chronic lymphocytic leukemia by multiplex PCR-based next generation sequencing. PLoS One. 2015;10(6) e0129544.

51. Athanasakis E, Melloni E, Rigolin GM, et al. The p53 transcriptional pathway is preserved in ATMmutated and NOTCH1mutated chronic lymphocytic leukemias. Oncotarget. 2014;5(24):12635-12645.

52. Rebay I, Fleming RJ, Fehon RG, et al. Specific EGF repeats of Notch mediate interactions with Delta and Serrate: impli- cations for notch as a multifunctional receptor. Cell. 1991;67(4):687-699.

53. Stanley P, Okajima T. Roles of glycosylation in notch signaling. Curr Top Dev Biol. 2010;92:131-164

54. Haines N, Irvine KD. Glycosylation regulates Notch signalling. Nat Rev Mol Cell Biol. 2003;4(10):786-797.

55.Pakkiriswami S, Couto A, Nagarajan U, Georgiou M. Glycosylated Notch and cancer. Front Oncol. 2016;6:37

56. Rand MD, Grimm LM, Artavanis-Tsakonas $S$, et al. Calcium depletion dissociates and activates heterodimeric notch receptors. Mol Cell Biol. 2000;20(5):1825-1835.

57. Rust R, Visser L, Van Der Leij J, et al. High expression of calcium-binding proteins, S100A10, S100A11 and CALM2 in anaplastic large cell lymphoma. Br J Haematol. 2005;131(5):596-608.

58. Dang Q, Chen L, Xu M, et al. The $\gamma$-secretase inhibitor GSI-I interacts synergistically with the proteasome inhibitor bortezomib to induce ALK+ anaplastic large cell lymphoma cell apoptosis. Cell Signal. 2019;59:76-84.

59. Hudson S, Wang D, Middleton F, et al. Crizotinib induces apoptosis and gene expression changes in ALK+ anaplastic large cell lymphoma cell lines; brentuximab synergizes and doxorubicin antagonizes. Pediatr Blood Cancer. 2018;65(8):e27094.

60. Larose H, Burke GAA, Lowe EJ, Turner SD From bench to bedside: the past, present and future of therapy for systemic paediatric ALCL, ALK. Br J Haematol. 2019;185 (6):1043-1054

61. Habets RA, de Bock CE, Serneels L, et al. Safe targeting of $\mathrm{T}$ cell acute lymphoblastic leukemia by pathology-specific NOTCH inhibition. Sci Transl Med. 2019;11(494): eaau6246.

62. Papayannidis C, DeAngelo DJ, Stock W, et al. A phase 1 study of the novel gammasecretase inhibitor PF-03084014 in patients with T-cell acute lymphoblastic leukemia and T-cell lymphoblastic lymphoma. Blood Cancer J. 2015;5:e350.

63. Samon JB, Castillo-Martin M, Hadler M, et al. Preclinical analysis of the $\gamma$-secretase inhibitor PF-03084014 in combination with glucocorticoids in T-cell acute lymphoblastic leukemia. Mol Cancer Ther. 2012;11(7): 1565-1575

64. Wei P, Walls M, Oiu M, et al. Evaluation of selective gamma-secretase inhibitor PF03084014 for its antitumor efficacy and gastrointestinal safety to guide optimal clinical trial design. Mol Cancer Ther. 2010;9(6): 1618-1628. 